\title{
Extracellular vesicles derived from mesenchymal stromal cells may possess increased therapeutic potential for acute kidney injury compared with conditioned medium in rodent models: A meta-analysis
}

\author{
GUANGYUAN ZHANG $^{1 *}$, DANDAN WANG ${ }^{2 *}$, SHUAI MIAO ${ }^{1 *}$, \\ XIANGYU ZOU ${ }^{1}$, GUOHUA LIU ${ }^{1}$ and YINGJIAN ZHU ${ }^{1}$ \\ ${ }^{1}$ Department of Urology, Shanghai First People's Hospital, School of Medicine, Shanghai Jiao Tong University, \\ Shanghai 200080; ${ }^{2}$ Department of Nephrology, The Second People's Hospital of Shenzhen, \\ Guangzhou Medical University, Shenzhen, Guangzhou, Guangdong 510182, P.R. China
}

Received December 4, 2014; Accepted January 26, 2016

DOI: $10.3892 /$ etm.2016.3076

\begin{abstract}
The potential involvement of the endocrine/paracrine mechanisms in the mesenchymal stromal cells (MSCs) therapy for acute kidney injury (AKI) has been increasingly studied. The aim of the present meta-analysis was to systematically review the therapeutic role of MSC-conditioned medium (CM) or MSCs released by extracellular vesicles (Evs) for the treatment of AKI in rodent models. Studies were identified using PubMed and Scopus databases using a custom search strategy and eligibility criteria. Data regarding serum creatinine ( $\mathrm{SCr}$ ) concentration, CM or Evs, measurement time point, AKI model (toxic or non-toxic) and other parameters, including delivery route, animal type and animal numbers, were extracted. Pooled analysis and subgroup analysis as well as multivariable meta-regression were performed. Heterogeneity and publication bias were also investigated. A total of 13 studies were included and analyzed. Pooled analysis showed reduced $\mathrm{SCr}$ (0.93 [0.67, $1.20], \mathrm{mg} / \mathrm{dl}$ ) in rodent models of AKI after CM/Evs therapy. The results of the subgroup analysis suggested that Evs induced an increased therapeutic effect, in the form of SCr reduction, as compared with $\mathrm{CM}(\mathrm{P}=0.05)$. There were also other significant influential factors for $\mathrm{SCr}$ reduction including measurement time point $(\mathrm{P}=0.0004)$ and therapeutic time point $(\mathrm{P}<0.0001)$ after surgery. By contrast, parameters such as delivery route, injury type and cell type were not significant influential factors.
\end{abstract}

Correspondence to: Dr Yingjian Zhu, Department of Urology, Shanghai First People's Hospital, School of Medicine, Shanghai Jiao Tong University, 100 Haining Road, Shanghai 200080, P.R. China E-mail: zhuyingjian_sjtu@126.com

*Contributed equally

Key words: mesenchymal stromal cells, conditioned medium, extracellular vesicles, acute kidney injury, meta-analysis
Multivariable meta-regression analysis showed that measurement time point $(\mathrm{P}=0.041)$, therapeutic time point $(\mathrm{P}=0.03)$, Evs or $\mathrm{CM}(\mathrm{P}=0.0003)$ and cell type $(\mathrm{P}<0.0001)$ were influential factors in the reduction of SCr. The present meta-analysis indicates that $\mathrm{CM}$ or Evs derived from MSCs are able to improve the impaired renal function in rodents modelling AKI. Compared with CM, Evs may produce a more marked therapeutic effect in recovery from renal failure. In addition, $\mathrm{CM}$ or Evs administration in early stages of AKI may result in more evident effects.

\section{Introduction}

Acute kidney injury (AKI) refers to a clinical syndrome characterized by a rapid (hours to days) reduction in renal excretory function, with the accumulation of creatinine and urea nitrogen and other waste products that are not commonly tested in clinical practice (1). AKI is commonly observed in clinical practice, particularly following major surgery and treatment in intensive care units (2). In addition, AKI mortality is high, ranging between 24 and $62 \%$ (3). Patients that survive AKI may have an increased long-term risk of developing chronic kidney disease with poor prognosis (4). There is therefore an urgent requirement for novel methods for the prevention and management of AKI.

In recent years, a promising and effective therapeutic strategy for AKI involves the use of mesenchymal stromal cells (MSCs) derived from various sources, such as bone marrow or adipose $(5,6)$. However, the mechanisms are not understood well. It has been suggested that MSCs promote renal injury repair, predominantly via paracrine/endocrine mechanisms as opposed to direct transdifferentiation into kidney cells $(7,8)$. In previous studies, MSC-conditioned medium and MSCs released by extracellular vesicles (Evs) were reported to exert renoprotective effects against AKI (9-11). The researchers attributed these effects to the favorable molecules, such as mRNA and miRNA (11), in the Evs or secreted soluble factors, such as hepatic growth factor. However, contradictory findings have 
indicated that $\mathrm{CM}$ may not be able to protect against kidney injury $(12,13)$.

EVs, such as exosomes and shedding vesicles (also known as microvesicles; Mvs) are membranous structures that deliver bioactive molecular content, including proteins, mRNA and micro (mi)RNA sequences (14). Evs is a term suggested in recent years which describes a novel pathway of cell-to-cell interaction, and it is also regarded as a crucial point of endocrine (14). Since Evs are released by cells and extracted from $\mathrm{CM}$ using differential centrifugation, in the present study Evs were regarded as a type of 'special CM' or 'improved CM'. On the basis of endocrine/paracrine mechanism of MSCs, CM/Evs could provide a novel strategy of cell-free therapy for tissue injuries (15).

Previous studies have produced inconsistent results regarding the effects of $\mathrm{CM} / \mathrm{Evs}$ therapy on $\mathrm{AKI}$ in rodent models $(8,12)$. This may be due to the variation of injury models, treatment models, delivery route and cell type. In present study, a meta-analysis was conducted to identify relevant literature regarding $\mathrm{CM} /$ Evs therapy applied to $\mathrm{AKI}$ in rodent models, using the serum creatinine ( $\mathrm{SCr}$ ) concentration, the classic index of kidney function, as an analyzed parameter. In addition, this study was intended to investigate the possible influential factors for the therapeutic effects by sub-group and regression analysis.

\section{Materials and methods}

Search strategy. PubMed (http://www.ncbi.nlm.nih. gov/pubmed) and Scopus (http://www.scopus.com) were employed as searched databases. The last search was updated on October 1st, 2014 using the following key words and search terms: ([extracellular vesicles or Evs or micro vesicles or micro-vesicles or microvesicles or Mvs or exosome or shedding vesicles] or [conditioned medium or conditioned culture media]) and (mesenchymal stromal cells or mesenchymal stem cells or MSCs) and (acute kidney injury or acute kidney disease or acute renal failure).

Eligibility criteria and data extraction. AKI models in rats or mice were screened. The animal experiments that investigated the effect of MSC-derived CM/Evs therapy on impaired renal function as determined by the level of $\mathrm{SCr}$ were analyzed. In addition, a sham- or placebo-operated control group was a requirement for study inclusion. The exclusion criteria for the studies were the following: i) Large animal/non-rodent experiments; ii) renal function was not determined by $\mathrm{SCr}$; iii) $\mathrm{SCr}$ estimation was not included; iv) cell behavior was altered by genetic modification. Comments, reviews, and editorials were excluded. Only published English-language studies were considered for inclusion.

The following data were extracted from the complete manuscripts of the qualified studies: Basal characteristics of the study, SCr concentration, CM or Evs therapy, time of the therapy after injury and measurement time. If necessary, $\mathrm{SCr}$ data were estimated using graphics, as previously described $(16,17)$. Accordingly, standard deviations were determined or recalculated based on the standard error. $\mathrm{SCr}$ concentrations are expressed herein as $\mathrm{mg} / \mathrm{dl}$ (original data presented as $\mu \mathrm{mol} / 1$ were changed accordingly). All literature searching, screening and data extraction were performed by two independent individuals, and determined after discussion.

Data analysis. The outcome was presented in teh form of the different in mean SCr between the control (AKI) and experimental (CM/Evs therapy) animals. A random-effects model was applied according to the results of heterogeneity tests. Continuous variables are presented as weighted mean differences with 95\% confidence intervals (CIs) between the MSC-treated and control groups. In the case of multiple experimental groups compared with one control group within a single study, the number of animals in the control group was divided equally by the number of experimental groups. When there were multiple measurements, one study was regarded as separate assessment $(16,17) . \mathrm{P}<0.05$ was considered to indicate a statistically significant difference in two-sided tests.

Sub-group analysis and multivariate meta-regression were performed. The analyzed influential factors included: CM or Evs; injury type, toxic or ischemia-reperfusion (IRI); cell type, bone marrow MSC (BMSC) or non-BMSC; delivery route, intravenous or others; time point of therapy after injury, $<1,1-24$ and $>24 \mathrm{~h}$; and time point of measurement, $\leq 2,3-4$ and $>4$ days. Furthermore, as publication bias is of concern for present meta-analysis, publication bias was investigated by a funnel plot.

All analysis was performed using Review Manager (version 5.2.9; The Nordic Cochrane Centre, Cochrane Collaboration, 2012) and SPSS software, version 18.0 (SPSS, Inc., Chicago, IL, USA). Meta-regression analysis was conducted using the 'MetaReg' macro written by David B. Wilson (http://mason.gmu.edu/ dwilsonb/ma.html).

\section{Results}

Literature characteristics. A total of 45 studies were retrieved from the PubMed database and 254 from the Scopus database. After excluding duplicate studies, a total of 274 remained. By excluding 96 review articles, 17 books, 14 book chapters and 4 short surveys, a total of 143 research articles remained. After screening for inclusion eligibility based on reading titles and abstracts, there were 13 papers eligible for our review. Among the included animals, only 461 animals met the inclusion criteria and were analyzed. Characteristics of the enrolled studies are described in Table I.

Meta-analysis. SCr data were continuous, as shown by the mean and standard deviation. Pooled analysis showed a $\mathrm{SCr}$ reduction of $0.93 \mathrm{mg} / \mathrm{dl}(95 \% \mathrm{CI}, 0.67-1.20 \mathrm{mg} / \mathrm{dl})$ in the $\mathrm{CM} /$ Evs therapy groups, as compared with the control groups with significant heterogeneity $\left(\mathrm{P}<0.00001 ; \mathrm{I}^{2}=96 \%\right.$; Fig. 1). Overall, no significant difference in $\mathrm{SCr}$ at baseline between the control and therapy groups was detected $(\mathrm{P}=0.83)$. In addition, several subgroup analyses were performed in order to determine whether $\mathrm{CM}$ or Evs have comparable therapeutic effects, and the optimum choice in CM/Evs therapy time after injury, time point measurement, delivery route, cell type and animal species.

The multivariable meta-regression analysis showed that measurement time point $(\mathrm{P}=0.041)$, therapeutic time point $(\mathrm{P}=0.03)$, Evs or $\mathrm{CM}(\mathrm{P}=0.0003)$ and cell type $(\mathrm{P}<0.0001)$ were independent influential factors of $\mathrm{SCr}$ reduction. 


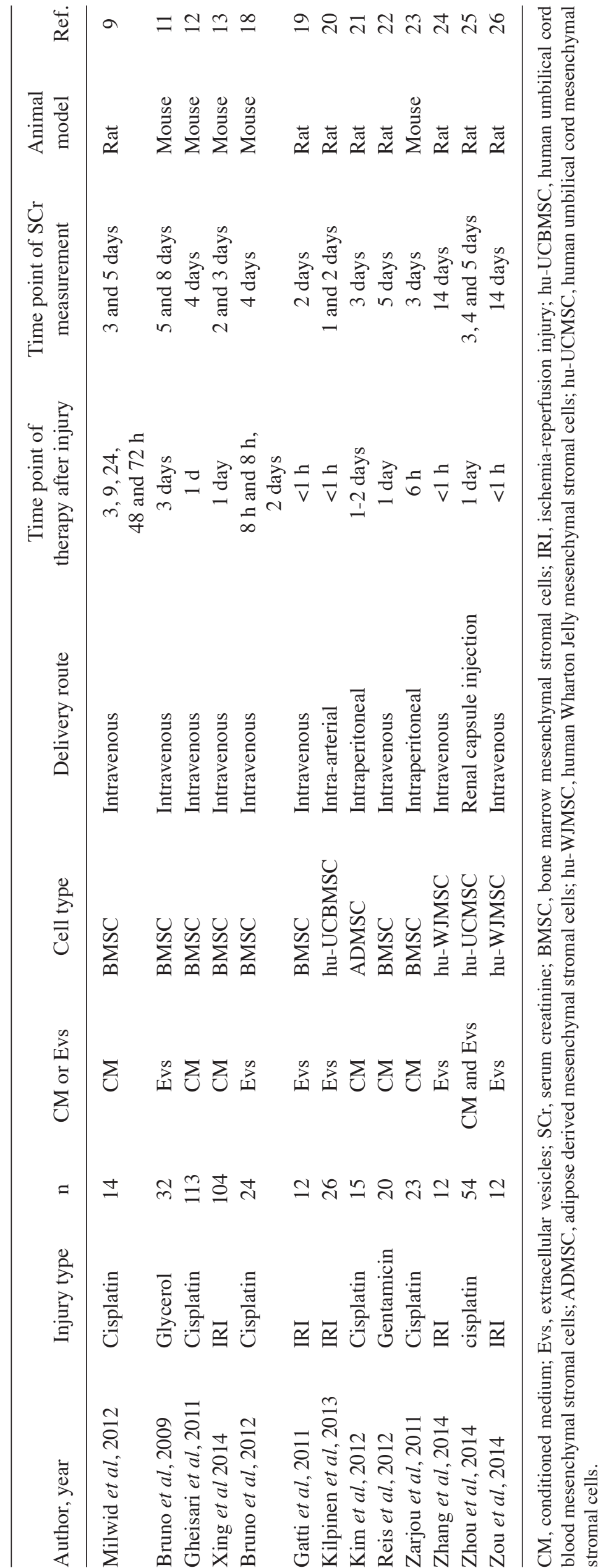




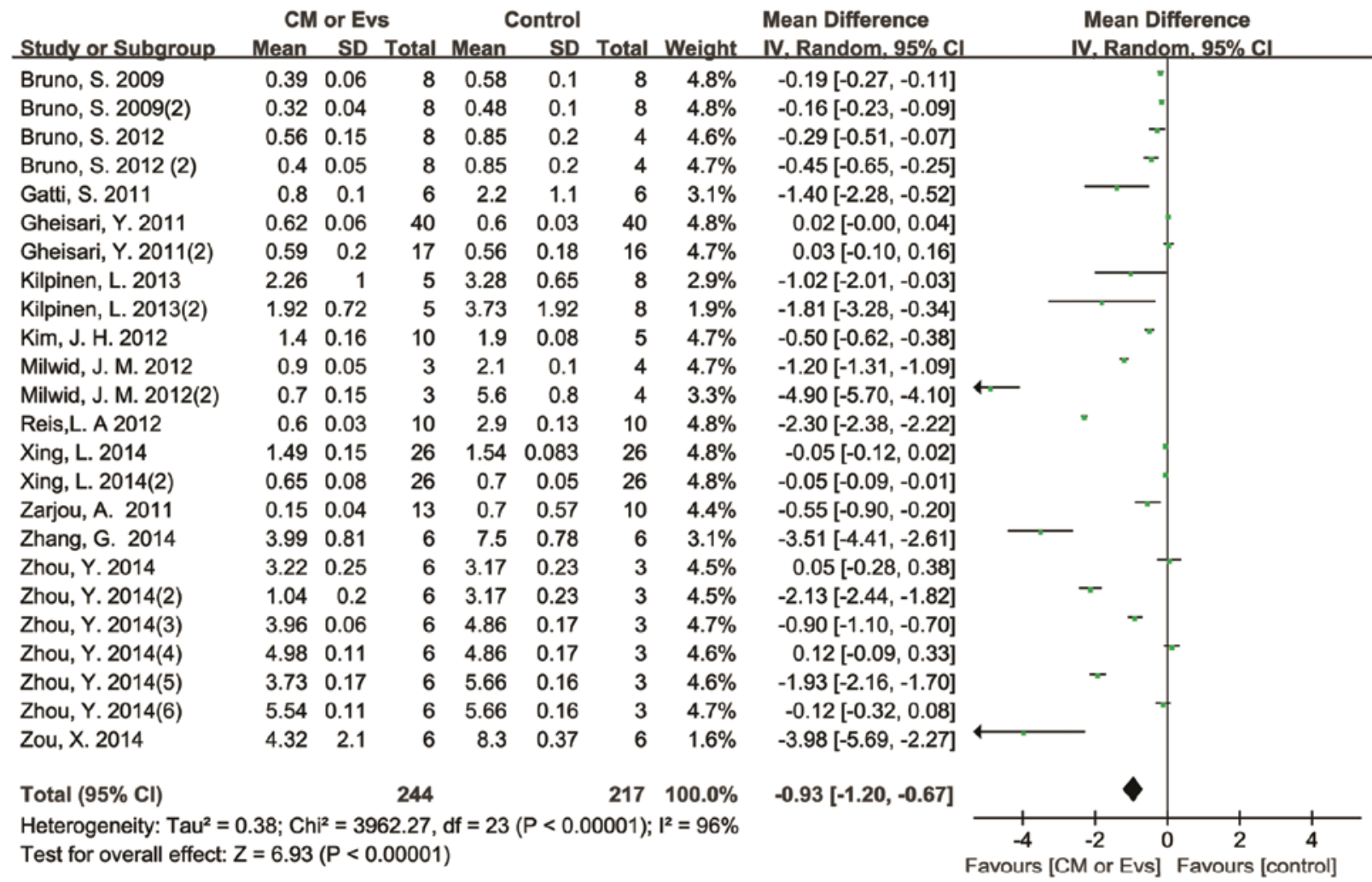

Figure 1. Forest plot shows the impact of CM/Evs derived from mesenchymal stromal cell injection on serum creatinine reduction compared with controls, 95\% CI: 95\% confidence interval. CM, conditioned medium; Evs, extracellular vesicles; SD, standard deviation; IV, inverse variance; CI, confidence interval.
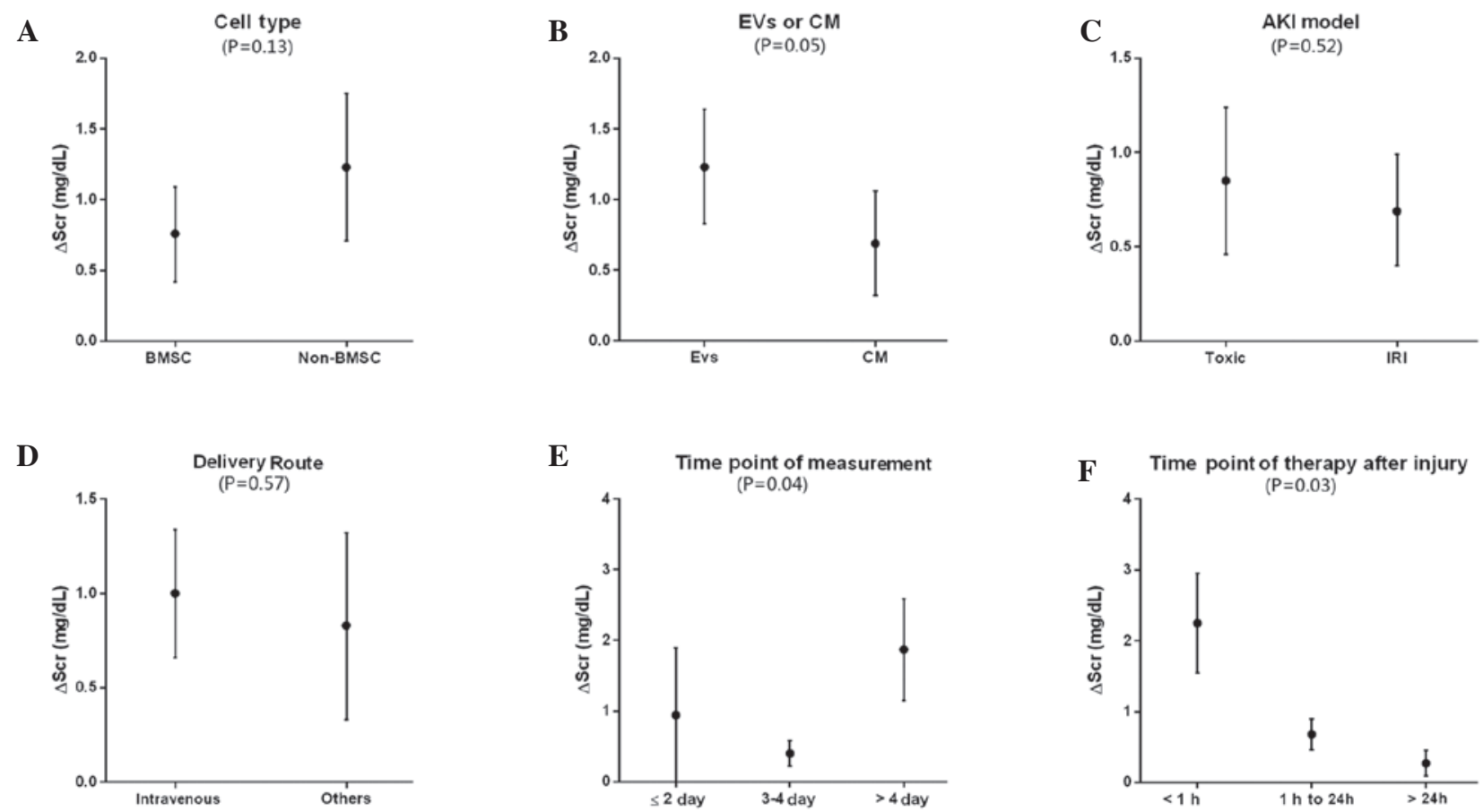

Figure 2. Sub-group analysis of influential factors for $\mathrm{SCr}$ reduction ( $\triangle \mathrm{SCr}$ in the figure). (A) SCr reduction from BMSC-treated and non-BMSC-treated sub-groups. (B) SCr reduction from EVs-treated and CM-treated subgroups. (C) SCr reduction from Toxic-AKI model and IRI-AKI sub-groups. (D) SCr reduction from intravenous treated and other delivery sub-groups. (E) SCr reduction from different time points of SCr mearsurement. (F) SCr reduction from different time points of therapy after injury. P-value was calculated using $\chi^{2}$ test for sub-group difference. BMSC, bone marrow mesenchymal stromal cells; Evs, extracellular vesicles; CM, conditioned medium; AKI, acute kidney injury; IRI, ischema-reperfusion injury.

In the sub-group analysis, no difference in $\mathrm{SCr}$ reduction was detected between BMSC and non-BMSC therapy groups $(0.76 \mathrm{mg} / \mathrm{dl}[1.09,0.42]$ vs. $1.23 \mathrm{mg} / \mathrm{dl}[1.75,0.71]$ $\mathrm{P}=0.13$ ). (Fig. 2A). These results were inconsistent with the 


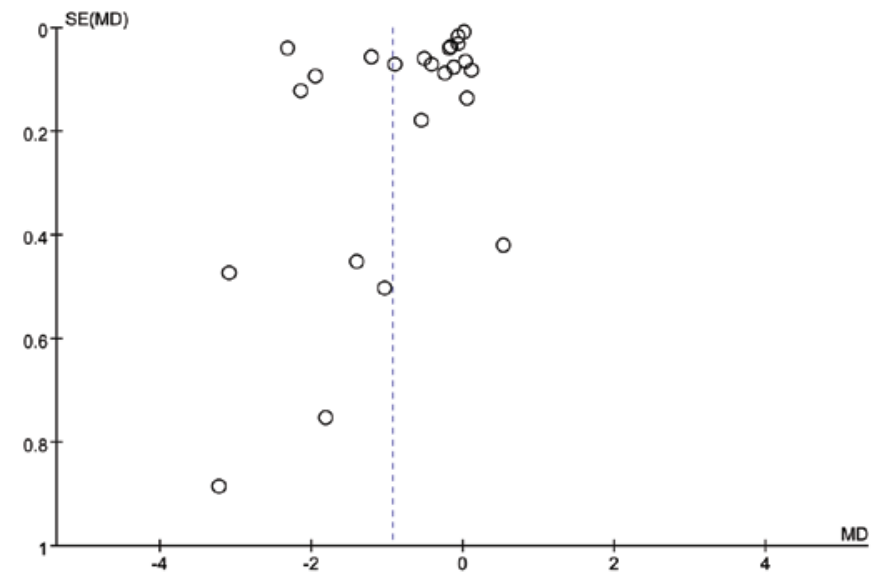

Figure 3. Funnel plot for serum creatinine reduction. Dotted line indicates the overall estimated mean difference. No obvious evidence for publication bias was detected. MD, mean difference; SE, standard error.

result of meta-regression, which indicated that cell type was an influential factor for SCr reduction. The sub-group analysis also detected significant differences between Evs and $\mathrm{CM}(\mathrm{P}=0.05)$, which showed the $\mathrm{SCr}$ reduction $(0.7 \mathrm{mg} / \mathrm{dl}$, [0.32,1.07] vs. $1.23 \mathrm{mg} / \mathrm{dl},[0.84,1.63]$, for Evs and CM respectively; Fig. 2B). Furthermore, the $\mathrm{SCr}$ reduction induced by Evs $(1.23 \mathrm{mg} / \mathrm{dl},[0.84,1.63])$ was increased compared with CM $(0.7 \mathrm{mg} / \mathrm{dl},[0.32,1.07])$. In the present study, AKI model or CM/Evs delivery route were not identified to be associated with $\mathrm{SCr}$ reduction (Fig. 2C and D). As shown in Fig. 2E, SCr measurement at $>4$ days after therapy was associated with more favorable effects $(1.87 \mathrm{mg} / \mathrm{dl},[1.14,2.59])$, while measurement at $\leq 2$ days showed no beneficial effects $(-0.94 \mathrm{mg} / \mathrm{dl},[-1.89,-0.00])$. Significant differences were also detected in the sub-group analysis $(\mathrm{P}=0.041)$. In addition, the therapeutic time point was an influential factor for $\mathrm{SCr}$ reduction (Fig. 2F). It was observed that CM/Evs injected within $1 \mathrm{~h}$ after injury were associated with a favorable outcome $(2.25 \mathrm{mg} / \mathrm{dl},[2.95,1.55])$, while at 1 day after injury the therapeutic effects were reduced $(0.27 \mathrm{mg} / \mathrm{dl},[0.09,0.45])$. The subgroup analysis demonstrated the significant differences associated with treatment time point $(\mathrm{P}=0.03)$. In present study, two species of animal were investigated, namely rats and mice. The sub-group analysis showed no significant difference between these animals $(\mathrm{P}=0.72)$, which was consistent with the meta-regression analysis.

As shown in Fig. 3, the funnel plot for $\mathrm{SCr}$ indicated no publication bias.

Sensitivity analysis. Sub-group and multivariable meta-analyses were performed to investigate the source of significant heterogeneity among the involved studies.

Analyzed factors included CM or Evs, injury type, animal type, cell type, delivery route, therapeutic time point (after injury) and measurement time point. Meta-regression showed that measurement time point $(\mathrm{P}=0.041)$, therapeutic time point $(\mathrm{P}=0.03)$, Evs or $\mathrm{CM}(\mathrm{P}=0.0003)$ and cell type $(\mathrm{P}<0.0001)$ were independent influential factors of $\mathrm{SCr}$ reduction. No trend in $\mathrm{SCr}$ reduction was observed regarding animal model $(\mathrm{P}=0.72)$.

\section{Discussion}

At present, adult stem cells have been extensively investigated with regard to their potential implications in regenerative medicine (27). MSCs from various tissues have been applied to the therapy for kidney injury, ischemia myocardial infarction and other diseases in clinical trials, a number of which produced favorable results (28). However, there remain a number of limitations associated with MSC transplantation, including immune-mediated rejection, senescence-induced genetic instability or loss of function and limited cell survival (29). Besides these issues, the primary problem related to the use of MSCs in clinical applications is the possibility of malignant transformation (30). On the basis of the endocrine/paracrine mechanism that may be involved in MSC therapy, CM/Evs may offer a strategy which avoids a number of the risks and limitations mentioned above (15).

The present meta-analysis comprised 13 published studies concerning on $\mathrm{CM} / \mathrm{Ev}$ for $\mathrm{AKI}$, and the pooled analysis showed a more marked $\mathrm{SCr}$ reduction $(0.93 \mathrm{mg} / \mathrm{dl}[0.67,1.20])$ in $\mathrm{CM} /$ Evs therapy groups compared with control groups, suggesting that $\mathrm{CM} / \mathrm{Evs}$ were able to protect rodent model animals against AKI. The sub-group analysis showed that $\mathrm{CM}$ and Evs administration could lead to $\mathrm{SCr}$ reduction (0.7 mg/dl [0.32,1.07] and $1.23 \mathrm{mg} / \mathrm{dl}$ [0.84,1.63], respectively). Furthermore, $\mathrm{SCr}$ reduction in Evs sub-group was significantly elevated compared with the $\mathrm{CM}$ sub-group $(\mathrm{P}=0.05)$. Thus, Evs may offer more substantial therapeutic effects compared with CM. After the long-time concerning on growth factors and cytokines which is an important part of the cellular secretome, it now appears that the cells secreted Evs instead of soluble factors, which has previously been regarded as the main cellular secretome with a more important function. Evs, including exosomes and shedding vesicles, have been shown to deliver genetic information and functional proteins as well as bioactive membrane. Previous studies have attributed the therapeutic effects of Evs to their role in cell-to-cell communication (14) or the capability to reprogram injured cells (31). Evs can be extracted from CM in vitro using differential centrifugation, although the protocol may vary between studies. Thus, we hypothesize that the more marked protective role of Evs may be attributed to higher concentration of effective ingredient, such as functional protein, mRNA, miRNA and DNA, in Evs compared with CM.

The sub-group analysis showed that the rapid delivery of $\mathrm{CM} / \mathrm{Evs}$ (1 $\mathrm{h}$ after injury) may lead to greater $\mathrm{SCr}$ reduction (Fig. 2F), and the therapeutic effects may emerge after 4 days (Fig. 2E), while there was no significant $\mathrm{SCr}$ reduction after 2 days. Furthermore, the review data suggested that the delivery route and kidney injury type might not affect $\mathrm{SCr}$ reduction. Notably, in a previous meta-analysis concerning MSCs therapy for impaired renal function in small animal models (16), increased $\mathrm{SCr}$ reduction was observed using an arterial delivery route compared with an intravenous route. For MSCs transplantation, intravenously delivered cells were retained in the lung capillaries (32), while intra-arterial delivery may lead to more efficient infusion. This may explain why arterial injection therapy is able to produce improved treatment effects. By contrast, no retained cells were detected in the lung capillaries after intravenous injection in CM/Evs therapy (11). In addition, 
Evs were able to migrate toward injured tissue, thus functioning in a similar manner to MSCs (15). Therefore, the results mentioned above indicate that delivery route may not affect the therapeutic efficacy of an Evs-based treatment for AKI.

Thus far, cell-free therapy using CM/Evs for AKI experiments have been performed only in small animals. Therefore, further animal experiments involving different species are necessary in order to assess the safety and efficiency of CM/Evs therapy, prior to human clinical trials. Meta-analysis of animal studies was not common, yet they were recommended in several settings (33-35), and could often guide research (36), even clinical endeavors. Based on the present meta-analysis, our recommendations for MSCs cell-free MSCs therapy (CM/Evs) for AKI are as follows: i) Compared with CM, Evs have the priority as they possess greater therapeutic potential; ii) the time point of treatment should be as early as possible after injury; iii) the therapeutic effects may emerge at a later time; and iv) the delivery route could not affect the therapeutic effects.

However, there were still limitations of present study. The limitation of meta-analysis is well known (37), our analysis was based on study outcomes, and we did not have access to individual data. Another limitation is that some data were estimated using graphics during data extraction. Besides, there was significant heterogeneity, which might be due to other unknown influential factors varied in the included studies. Nevertheless, by using the random-effect analysis, the risk of finding erroneous estimates was minimized.

\section{Acknowledgements}

This study was supported by grants from the Research Program of Science and Technology Commission of Shanghai Municipality (grant no. 10411967200), Shanghai å Health Bureau (grant no. 2011PD06), National Natural Science Foundation of China (grant nos. 81170642 and 81470919) and a Shanghai Shen Kang Platform Grant (grant no. SHDC12007206). The authors thank Dr Changxin Song of Qinghai Normal University for consultation of statistical analysis.

\section{References}

1. Bellomo R, Kellum JA and Ronco C: Acute kidney injury. Lancet 380: 756-766, 2012

2. Bagshaw SM, George C and Bellomo R; ANZICS Database Management Committee: Early acute kidney injury and sepsis: A multicentre evaluation. Crit Care 12: R47, 2008.

3. Waikar SS, Liu KD and Chertow GM: Diagnosis, epidemiology and outcomes of acute kidney injury. Clin J Am Soc Nephrol 3: 844-861, 2008.

4. Wald R, Quinn RR, Luo J, Li P, Scales DC, Mamdani MM and Ray JG; University of Toronto Acute Kidney Injury Research Group: Chronic dialysis and death among survivors of acute kidney injury requiring dialysis. JAMA 302: 1179-1185, 2009.

5. Qian H, Yang H, Xu W, Yan Y, Chen Q, Zhu W, Cao H, Yin Q, Zhou H, Mao F and Chen Y: Bone marrow mesenchymal stem cells ameliorate rat acute renal failure by differentiation into renal tubular epithelial-like cells. Int J Mol Med 22: 325-332, 2008.

6. Gao J, Liu R, Wu J, Liu Z, Li J, Zhou J, Hao T, Wang Y, Du Z, Duan $C$ and Wang C: The use of chitosan based hydrogel for enhancing the therapeutic benefits of adipose-derived MSCs for acute kidney injury. Biomaterials 33: 3673-3681, 2012.

7. Duffield JS, Park KM, Hsiao LL, Kelley VR, Scadden DT, Ichimura T and Bonventre JV: Restoration of tubular epithelial cells during repair of the postischemic kidney occurs independently of bone marrow-derived stem cells. J Clin Invest 115: 1743-1755, 2005.
8. Tögel F, Hu Z, Weiss K, Isaac J, Lange C and Westenfelder C: Administered mesenchymal stem cells protect against ischemic acute renal failure through differentiation-independent mechanisms. Am J Physiol Renal Physiol 289: F31-F42, 2005.

9. Milwid JM, Ichimura T, Li M, Jiao Y, Lee J, Yarmush JS, Parekkadan B, Tilles AW, Bonventre JV and Yarmush ML: Secreted factors from bone marrow stromal cells upregulate IL-10 and reverse acute kidney injury. Stem Cells Int 2012: 392050, 2012.

10. Bi B, Schmitt R, Israilova M, Nishio H and Cantley LG: Stromal cells protect against acute tubular injury via an endocrine effect. J Am Soc Nephrol 18: 2486-2496, 2007.

11. Bruno S, Grange C, Deregibus MC, Calogero RA, Saviozzi S, Collino F, Morando L, Busca A, Falda M, Bussolati B, et al: Mesenchymal stem cell-derived microvesicles protect against acute tubular injury. J Am Soc Nephrol 20: 1053-1067, 2009.

12. Gheisari Y, Ahmadbeigi N, Naderi M, Nassiri SM, Nadri S and Soleimani M: Stem cell-conditioned medium does not protect against kidney failure. Cell Biol Int 35: 209-213, 2011.

13. Xing L, Cui R, Peng L, Ma J, Chen X, Xie RJ and Li B: Mesenchymal stem cells, not conditioned medium, contribute to kidney repair after ischemia-reperfusion injury. Stem Cell Res Ther 5: 101, 2014.

14. Camussi G, Deregibus MC, Bruno S, Cantaluppi V and Biancone L: Exosomes/microvesicles as a mechanism of cell-to-cell communication. Kidney Int 78: 838-848, 2010.

15. Baglio SR, Pegtel DM and Baldini N: Mesenchymal stem cell secreted vesicles provide novel opportunities in (stem) cell-free therapy. Front Physiol 3: 359, 2012.

16. Wang Y, He J, Pei X and Zhao W: Systematic review and meta-analysis of mesenchymal stem/stromal cells therapy for impaired renal function in small animal models. Nephrology (Carlton) 18: 201-208, 2013.

17. van der Spoel TI, Jansen of Lorkeers SJ, Agostoni P, van Belle E, Gyöngyösi M, Sluijter JP, Cramer MJ, Doevendans PA and Chamuleau SA: Human relevance of pre-clinical studies in stem cell therapy: Systematic review and meta-analysis of large animal models of ischaemic heart disease. Cardiovasc Res 91: 649-658, 2011.

18. Bruno S, Grange C, Collino F, Deregibus MC, Cantaluppi V, Biancone L, Tetta $\mathrm{C}$ and Camussi G: Microvesicles derived from mesenchymal stem cells enhance survival in a lethal model of acute kidney injury. PLoS One 7: e33115, 2012.

19. Gatti S, Bruno S, Deregibus MC, Sordi A, Cantaluppi V, Tetta C and Camussi G: Microvesicles derived from human adult mesenchymal stem cells protect against ischaemia-reperfusion-induced acute and chronic kidney injury. Nephrol Dial Transplant 26: 1474-1483, 2011.

20. Kilpinen L, Impola U, Sankkila L, Ritamo I, Aatonen M, Kilpinen S, Tuimala J, Valmu L, Levijoki J, Finckenberg P, et al: Extracellular membrane vesicles from umbilical cord blood-derived MSC protect against ischemic acute kidney injury, a feature that is lost after inflammatory conditioning. J Extracell Vesicles 2, 2013.

21. Kim JH, Park DJ, Yun JC, Jung MH, Yeo HD, Kim HJ, Kim DW, Yang JI, Lee GW, Jeong SH, et al: Human adipose tissue-derived mesenchymal stem cells protect kidneys from cisplatin nephrotoxicity in rats. Am J Physiol Renal Physiol 302: F1141-F1150, 2012.

22. Reis LA, Borges FT, Simões MJ, Borges AA, Sinigaglia-Coimbra R and Schor N: Bone marrow-derived mesenchymal stem cells repaired but did not prevent gentamicin-induced acute kidney injury through paracrine effects in rats. PLoS One 7: e44092, 2012.

23. Zarjou A, Kim J, Traylor AM, Sanders PW, Balla J, Agarwal A and Curtis LM: Paracrine effects of mesenchymal stem cells in cisplatin-induced renal injury require heme oxygenase-1. Am J Physiol Renal Physiol 300: F254-F262, 2011.

24. Zhang G, Zou X, Miao S, Chen J, Du T, Zhong L, Ju G, Liu G and Zhu Y: The anti-oxidative role of micro-vesicles derived from human wharton-jelly mesenchymal stromal cells through NOX2/gp91(phox) suppression in alleviating renal ischemia-reperfusion injury in rats. PLoS One 9: e92129, 2014.

25. Zhou Y, Xu H, Xu W, Wang B, Wu H, Tao Y, Zhang B, Wang M, Mao F, Yan Y, et al: Exosomes released by human umbilical cord mesenchymal stem cells protect against cisplatin-induced renal oxidative stress and apoptosis in vivo and in vitro. Stem Cell Res Ther 4: 34, 2013.

26. Zou X, Zhang G, Cheng Z, Yin D, Du T, Ju G, Miao S, Liu G, Lu M and Zhu Y: Microvesicles derived from human Wharton's Jelly mesenchymal stromal cells ameliorate renal ischemia-reperfusion injury in rats by suppressing CX3CL1. Stem Cell Res Ther 5: 40, 2014.

27. Fisher MB and Mauck RL: Tissue engineering and regenerative medicine: recent innovations and the transition to translation. Tissue Engineering Part B: Reviews 19: 1-13, 2013. 
28. Wei X, Yang X, Han ZP, Qu FF, Shao L and Shi YF: Mesenchymal stem cells: A new trend for cell therapy. Acta Pharmacol Sin 34: 747-754, 2013.

29. Lim PK, Bliss SA, Patel SA, Taborga M, Dave MA, Gregory LA, Greco SJ, Bryan M, Patel PS and Rameshwar P: Gap junction-mediated import of microRNA from bone marrow stromal cells can elicit cell cycle quiescence in breast cancer cells. Cancer Res 71: 1550-1560, 2011.

30. Rubio D, Garcia S, Paz MF, De la Cueva T, Lopez-Fernandez LA, Lloyd AC, Garcia-Castro J and Bernad A: Molecular characterization of spontaneous mesenchymal stem cell transformation. PLoS One 3: e1398, 2008.

31. Camussi G, Deregibus MC and Cantaluppi V: Role of stem-cell-derived microvesicles in the paracrine action of stem cells. Biochem Soc Trans 41: 283-287, 2013.

32. Du T, Cheng J, Zhong L, Zhao XF, Zhu J, Zhu YJ and Liu GH: The alleviation of acute and chronic kidney injury by human Wharton's jelly-derived mesenchymal stromal cells triggered by ischemia-reperfusion injury via an endocrine mechanism. Cytotherapy 14: 1215-1227, 2012.
33. Sandercock P and Roberts I: Systematic reviews of animal experiments. Lancet 360: 586, 2002.

34. Horn J, De Haan R, Vermeulen M, Luiten P and Limburg M: Nimodipine in animal model experiments of focal cerebral ischemia: A Systematic Review. Stroke 32: 2433-2438, 2001.

35. Roberts I, Kwan I, Evans P and Haig S: Does animal experimentation inform human healthcare? Observations from a systematic review of international animal experiments on fluid resuscitation. BMJ 324: 474-476, 2002.

36. Biondi-Zoccai GG, Abbate A, Parisi Q, Agostoni P, Burzotta F, Sandroni C, Zardini P, Biasucci LM: Is vasopressin superior to adrenaline or placebo in the management of cardiac arrest? A meta-analysis. Resuscitation 59: 221-224, 2003.

37. Verstraete M: Value and limitation of meta-analysis. Pathophysiol Haemost Thromb 32: 278-281, 2002. 